\title{
Sarid Yishaï, Le monstre de la mémoire
}

\section{Sophie Lenis}

\section{OpenEdition}

\section{Journals}

Édition électronique

URL : https://journals.openedition.org/tsafon/3013

DOI : $10.4000 /$ tsafon. 3013

ISSN : 2609-6420

\section{Éditeur}

Association Jean-Marie Delmaire

\section{Édition imprimée}

Date de publication : 1 juillet 2020

Pagination : 185-186

ISSN : $1149-6630$

\section{Référence électronique}

Sophie Lenis, « Sarid Yishaï, Le monstre de la mémoire », Tsafon [En ligne], 79 | 2020, mis en ligne le 01 juillet 2020, consulté le 27 juin 2021. URL : http://journals.openedition.org/tsafon/3013 ; DOI : https:// doi.org/10.4000/tsafon.3013 


\section{À travers les livres .......}

Sarid Yishaï, Le monstre de la mémoire, Arles, Actes Sud, 2020, traduit de l'hébreu par Laurence Sendrowicz ( 1 ère éd. En hébreu, 2017), 160 p., 18,50€.

Plutôt habitué à l'écriture de polars, l'avocat et écrivain israélien Yishaï Sarid propose avec Le monstre de la mémoire un roman d'une toute autre facture. Sous la forme d'une lettre adressée au président de Yad Vashem «représentant officiel de la mémoire » (p. 6), le narrateur explique comment, jeune homme aspirant à se construire une situation professionnelle stable et confortable, une vie rangée à l'abri des regards, son esprit tourmenté a sombré dans un abîme incommensurable, proche de la folie.

Après un échec au concours du ministère des Affaires étrangères l'obligeant à renoncer à une carrière de diplomate, il s'oriente vers des études d'histoire. Encouragé par ses brillants résultats de jeune historien en devenir et intéressé par une spécialisation dans l'histoire de l'Asie et de l'Extrême-Orient, il dut très vite y renoncer, ne maitrisant pas les langues orientales. Désormais marié, installé en Israël et poussé par des contingences matérielles, il dut accepter de « faire une thèse en rapport avec la Shoah un sujet effrayant pour [lui] qui voulait mener [sa] barque qu'en eaux calmes, loin des tensions et des émotions fortes » (p. 11).

Dans le cadre de son sujet de doctorat «Étude comparative des méthodes d'extermination mises en œuvre dans les camps de la mort », il accepta de se confronter au «devoir de mémoire» (p. 12). Résigné, mais néanmoins soucieux de réussir, il emmagasina frénétiquement une très grande quantité d'informations sur l'histoire de la Shoah. Reconnu par ses pairs, il devint guide à Yad Vashem et le ministère de l'Éducation nationale lui confia la charge d'accompagner les classes de terminale, une semaine en Pologne, dans les camps de la mort. Il faut entendre ici les quatre centres de mise à mort: Chelmno, Belzec, Treblinka et Sobibor et les deux camps de concentration-extermination, dits mixtes que sont Majdanek et Auschwitz-Birkenau. Mû au départ par une certaine «extase intellectuelle» (p. 19), il se heurte très vite à l'indifférence de cette jeunesse enveloppée du drapeau national, des kippas sur la tête et dont il déplore toutes les "mièvreries ritualisées » comme celles des jeunes Israéliens qui ont, pour habitude, d'interpréter l'HaTikva, l'hymne israélien, et différents chants accompagnés à la guitare dans ces centres de mise à mort. Ils allument également des bougies et récitent le kaddish. Contaminé par le «virus de la mémoire » (p. 127) et la nécessité de transmettre, il cherche à choquer son auditoire, n'hésitant pas à entrer dans les moindres détails de la mise à mort, fasciné malgré lui par les bourreaux et par l'efficacité des méthodes de mise à mort.

Dans un style abrupt, dénué de compassion, le narrateur ne ménage personne, ni le Président à qui il s'adresse, ni le déporté qui l'accompagne, incapable de raconter sa propre histoire et de donner du sens à la visite de Birkenau, ni les élèves qu'ils dénigrent et avec lesquels ils n'arrivent pas à entrer en contact, ni sa propre personne mise à rude 
épreuve nerveusement par la répétition de cette histoire inlassablement, complétement engloutie par « le monstre de la mémoire».

Par cette fiction, l'auteur engage une réflexion profonde, voire dérangeante sur l'enseignement et la transmission de la mémoire de la Shoah. Comment assumer la mémoire d'un tel événement ? Quel message transmettre et comment ? La transmission de la mémoire doit-elle nécessairement passer par la visite de ces " lieux de haine » (p. 53) sans risquer la contagion? Le narrateur est persuadé que « la haine [pousse] sur les lieux de haine » et que les jeunes Israéliens s'emplissent de haine sur ces lieux « non pas envers les bourreaux, mais envers les victimes »(p. 53-54). Enfin, une réflexion plus générale sur la nature humaine taraude le narrateur qui, désespéré, proche du délire, en arrive à penser que « les êtres humains sont des assassins par essence » (p. 113).

Sophie Lenis

Birnbaum Pierre, La leçon de Vichy. Une histoire personnelle, Paris, Seuil, 2019, 240 p., $20 €$.

«Théoricien de l'État fort à la française » comme le définit la quatrième page de couverture, Pierre Birnbaum livre une histoire toute personnelle, son enfance de petit juif traqué et caché pendant la guerre, dans un récit qui tient largement compte du contexte et des événements historiques et politiques qui scandent la vie chaotique des juifs entre 1940 et 1945. Ceci explique sans doute le titre : «La leçon de Vichy », d'un livre qui expose deux histoires, celle de l'auteur certes mais aussi celle du Gouvernement de Vichy dont P.B. entend redéfinir la nature, ce qu'il n'avait jamais songé à faire avant qu'il ne revisite sa petite enfance.

C'est son ami Pierre Assouline qui l'a incité à s'engager dans ce parcours rétrospectif. Et il le fait en tant qu'historien. Les premiers chapitres rapportent les souvenirs et la mémoire, étayés par de nombreuses recherches méticuleuses en archives (nationales, départementales, du CDJC etc.), par la lecture d'essais et de travaux universitaires tels des mémoires de maîtrise sur l'histoire régionale des Hautes-Pyrénées, département où se niche, se dissimule dans les montagnes Omex, le village où Maria et Fabien, couple de paysans, le cachèrent sans se poser de questions : « ...c'est cette fois un pan de ma vie personnelle que j'expose imprudemment, c'est une plongée inattendue dans l'intime, les non-dits des cours ex cathedra, des livres et des articles des revues professionnelles », précise-t-il dès la p. 10.

La mémoire de son enfance se résume à Omex, non loin de Lourdes où il est né le 19 juillet 1940, en pleine déroute de la France à laquelle ses parents participent en fuyant vers le sud, vers l'Espagne proche. « Lourdes est une ville improbable pour la naissance d'un enfant juif, au terme d'un périple qui a mené mes parents dans le sud de la France depuis Varsovie et Dresde, en passant par Berlin », p. 20-21. Ce qui permet à la religieuse qui aide sa mère à accoucher de faire connaissance avec un bébé juif à propos duquel elle s'exclame : "Quel beau bébé, quel dommage que ce soit un Juif !» p. 21. $\mathrm{Ce}$ récit d'égo-histoire s'insère dans l'histoire des juifs cachés dans les HautesPyrénées, ce qui invite l'auteur à fouiller les fonds d'archives pour trouver un nombre important de documents concernant ses parents. Les grandes rafles en zone libre de l'été 1942 prennent une large part dans cette évocation tant elles anéantissent, chez les juifs réfugiés dans les départements pyrénéens, l'illusion d'une région montagnarde protectrice. Et les photos personnelles viennent compléter les reproductions de documents d'archives.

Après avoir retrouvé Omex, où se déroula une vie heureuse, champêtre, protégée par des familles taiseuses, et les tribulations d'une famille juive; le lecteur découvre les titres des chapitres III et IV qui parlent d'eux-mêmes : "L' « État français » m'a tué » et 
«Les Justes m'ont sauvé ». L'ancien professeur de Paris 1 retrouve alors son écriture pour donner les leçons sur : «Le président, l'État et la théorie de l'État » et « La fin de l'alliance royale ? », thèmes qui occupèrent bien des années de réflexion en sciences politiques et qui furent étudiés durant une carrière bien remplie d'universitaire. Et l'on perçoit à nouveau les critiques historiographiques sur ces sujets, abordés par un Rémond ou un Duverger, dépourvus eux d'un passé qui pouvait les sensibiliser à une démarche plus juste. Les deux derniers chapitres sont de véritables cours, des leçons, que l'on pourrait entendre dans un amphi de Sciences Po. Les démonstrations bien construites se faufilent au cours des pages et les références bibliographiques savantes ne manquent pas.

Ce livre est donc un savoureux mélange d'histoire mémorielle d'un enfant juif caché et d'exposé historique sur la nature de l'État en France et plus particulièrement sous le Gouvernement de Vichy.

Danielle Delmaire

Pinol Jean-Luc, Convois. La déportation des Juifs de France, Paris, éd. du Détour, septembre 2019, 319 p., 24,90€.

«Quand la cartographie aide à mieux comprendre l'histoire », tel pourrait être le soustitre de cet ouvrage qui rassemble pas moins de 130 cartes sur la déportation des Juifs de France. Il ne s'agit plus de lire des récits de témoins ou des analyses d'historiens mais de visualiser l'ampleur de la déportation, à l'échelle nationale et à l'échelle régionale, voire à l'échelle de la capitale : Paris.

Grâce aux cartes, élaborées à partir du Mémorial de la Déportation des Juifs de France de Serge Klarsfeld, accessible depuis 2012 en version numérique, les différentes manifestations de la persécution sont mises en évidence. Par exemple, les circonstances de l'arrestation varient selon le lieu d'habitation. Les cartes de Paris montrent que l'on n'est pas arrêté dans les mêmes circonstances si l'on réside dans les quartiers huppés de l'ouest parisien, là où vivent les juifs français depuis longtemps, ou dans les quartiers plus pauvres, là où se sont installés les familles juives immigrées récemment. Et pour ces familles juives immigrées, les arrestations ne sont pas exécutées dans les mêmes occasions selon que l'arrivée en France est très récente ou un peu plus ancienne. Dans tous les cas, les réseaux d'aide éventuelle ne sont pas identiques et la cartographie localise ces différences. De même, les cartes donnent une image plus globale des divers moyens d'arrestation selon qu'elle a lieu au domicile habituel ou à partir d'un refuge, ville, village ou institution. Ainsi surgit la complexité des «destins individuels » tout comme la complexité des « destins, familiaux ou collectifs ».

Apparaît aussi nettement la géographie de la déportation des juifs grâce aux cartes 1.1 et 1.2 : les arrestations pour la déportation ont été réalisées dans un dixième des 36000 communes que compte la France. Toutes les régions sont touchées mais il en est où les arrestations furent quasiment absentes : la Corse, la Bretagne et l'Alsace. Pour la région Nord-Pas-de-Calais, l'histoire se lit sur ces deux cartes où l'on distingue clairement la région lilloise, le bassin minier, depuis Lens jusqu'à Valenciennes, et le Cambrésis : c'est là où la rafle du 11 septembre 1942 fit le plus de ravages.

La visualisation de la déportation des juifs de France se fait aussi par graphiques, tel le graphique 2.1 qui donne les lieux de naissance des déportés selon chacun des 77 convois. Tous les lieux de naissance n'ont pas pu être listés, l'auteur se limite, fort justement, à quelques grandes villes: Paris, Strasbourg, Marseille et Oran pour la France et ses colonies, Francfort, Berlin et Vienne pour l'Europe d'expression allemande, Salonique et Constantinople pour la Méditerranée orientale, Anvers et Amsterdam pour l'Europe du Nord-Ouest et enfin Varsovie pour l'aire polonaise. Pour 
Paris, les petites colonnes du graphique sont plus hautes pour les convois d'août 1942, mettant en évidence la densité des départs dus à la rafle du Vel' d'Hiv. La rafle de Marseille des 22-24 janvier 1943 se lit avec l'allongement des colonnes de Marseille et d'Oran pour le convoi 52 du 23 mars 1943. La présence majoritaire des juifs nés à Varsovie dans les convois 1 à 16 (jusqu'au 7 août 1942) montre qu'ils furent bien les premières victimes de la déportation qui visait, dans un premier temps et prioritairement, les étrangers. Ce graphique est particulièrement éloquent, il est difficile d'en faire une analyse complète dans une brève recension.

Avec ce graphique, toujours, apparaît le calendrier de la déportation et ses différentes phases. Celles-ci sont présentées dans le chapitre trois, chacune groupant un peu moins ou un peu plus d'une dizaine de convois. Et bien sûr des cartes illustrent ces phases si bien que la géographie rejoint la chronologie. Ce chapitre intègre le convoi parti de Malines après la rafle du 11 septembre 1942 dans le Nord-Pas-de-Calais et l'auteur en souligne la particularité en évoquant un convoi « atypique par rapport à l'ensemble des convois partis de Drancy » (p. 72).

En réalité l'ouvrage outrepasse l'annonce du titre puisque le chapitre cinq donne la cartographie des « fusillés, guillotinés, massacrés », non déportés, avec les cartes des régions particulièrement touchées par les massacres, comme la Dordogne ou la région lyonnaise.

Une carte des Justes complète cet ensemble. L'auteur a soin de préciser qu'une telle carte ne peut rendre compte que partiellement des sauvetages dans la mesure où les dossiers de Yad Vashem sont constitués à partir des témoignages des sauvés. Si la personne secourue ne fait aucune démarche, son ou ses sauveteurs ne seront jamais honorés du titre de Justes parmi les Nations et le sauvetage reste inconnu.

Enfin dans les 80 pages d'annexes, se suivent les cartes de chacun des convois donnant les effectifs, la localisation des arrestations ou des rafles.

Quel ouvrage est parfait? Il faut donc émettre quelques reproches. Celui d'abord de confondre «zone rattachée » et «zone annexée » : la Moselle et les deux départements alsaciens ne sont pas « rattachés au Reich» (p. 21 et cartes p. 22-23) mais annexés, tandis que le Nord et le Pas-de-Calais sont rattachés au commandement militaire de Bruxelles. Il y a là une différence administrative importante pour le sort des populations en général et des juifs en particulier. Quant à la carte 1.8, p. 33, elle donne à voir les lieux où les juifs sont décédés dans des camps d'internement et dans des lieux de transit français. Aucune trace des camps du Boulonnais où pourtant quelques juifs sont décédés. Mais il est vrai que ces juifs étaient originaires de Belgique et non de France. Camps en France pour juifs de Belgique : à quelle histoire appartiennent-ils ? De France ou de Belgique? (Lire l'introduction au dossier).

Malgré ces rares écarts, il convient de conclure avec Serge Klarsfeld qui a préfacé l'ouvrage : « ce présent livre est un nouveau développement dans l'historiographie de la Shoah » (p. 5). Et ajoutons que J-L Pinol, professeur émérite de l'ENS de Lyon, a fourni un excellent outil pédagogique pour ses collègues de l'enseignement secondaire qui souhaitent focaliser leur cours sur l'histoire régionale, celle de leurs élèves, grâce à ces nombreuses cartes.

Rota Olivier, Apostolat catholique et travail social en milieu juif. Les Ancelles de Notre-Dame de Sion (1926-1964), Paris, éd. du Cerf, août 2019, 261 p., $26 €$.

O. Rota, désormais titulaire d'une habilitation à diriger des recherches, est l'auteur de nombreux ouvrages et articles sur les relations entre juifs et chrétiens, en France et en Angleterre (thème principal de sa soutenance d'HDR, novembre 2019). Il ajoute, à sa 
copieuse bibliographie, une œuvre qui devrait faire date dans l'histoire de l'apostolat de l'Église catholique envers les juifs.

Suite à des démêlés judiciaires pour avoir abusivement converti des juifs, les sœurs de Notre-Dame de Sion, dont la vocation était de sortir les juifs de leurs erreurs et leur aveuglement en les amenant à la conversion au catholicisme, remettent en cause, à la fin $\mathrm{du} \mathrm{XIX}^{\mathrm{e}}$ siècle, leur apostolat envers les juifs. Le but de la conversion n'est pas abandonné, tant les sœurs sont convaincues d'agir pour le bien des âmes juives, mais ce sont les méthodes qui sont revues et corrigées. Dans ce changement d'action apostolique, les Ancelles de Notre-Dame de Sion ont joué un rôle pionnier et primordial, durant une quarantaine d'années - parmi lesquelles s'écoulent les années de guerre et de persécution des juifs. C'est cette aventure entreprise par des femmes catholiques, croyantes et emplies d'empathie pour les juifs, qu'Olivier Rota expose dans ce livre.

L'auteur consacre ses premières pages à une rétrospective de l'œuvre des sœurs de Sion, ce qui permet de mieux percevoir l'évolution de la pensée sionienne. Quelques sœurs finissent par s'interroger sur la pertinence de leurs actions et par prendre conscience de la nécessité d'agir autrement que par la pression morale sur des familles, le plus souvent en détresse. La réflexion se met en place avant la Première Guerre mondiale et la mise en œuvre d'autres méthodes apostoliques apparaît dès les années 1920, privilégiant les prières. Amici Israël, bien que n'émanant pas de Notre-Dame de Sion, est une œuvre qui agit dans le même sens et influence plus ou moins quelques jeunes femmes sensibilisées à ce nouvel apostolat envers les juifs.

Ce sont donc des travailleuses sociales : assistantes sociales, infirmières, qui s'engagent sur ce terrain en pénétrant les milieux juifs, autant que possible, afin d'obtenir la sympathie de voisins juifs puis de leur servir de modèle de vie et les amener, doucement, lentement, à la conversion. Il ne s'agit pas de convertir en grand nombre et l'accent est mis sur la qualité et la sincérité de cette conversion.

Ces jeunes femmes s'installent donc là où les juifs se trouvent : en Palestine où elles fondent les Servantes de Notre-Dame Reine de Palestine, dans l'entre-deux-guerres, elles deviennent les Ancelles quand elles se rattachent à la congrégation des sœurs de Sion. Mais rattachement ne signifie pas complète intégration. En effet, les Ancelles revendiquent la possibilité de vivre en dehors de la congrégation, en petites communautés installées en ville et de mener un travail social, incognito, parmi les juifs, en refusant de porter l'habit. Elles rejoignent, me semble-t-il, la position d'une Madeleine Delbrêl, elle aussi assistante sociale qui œuvra dans un milieu déshérité, à la manière des prêtres ouvriers, un peu plus tard. Les Ancelles s'implantent également à Paris dans le quartier du Marais où, sans faire une grande publicité de leur appartenance au catholicisme, elles se dévouent pour aider des familles nécessiteuses et pour prendre en charge la scolarité d'enfants parfois livrés à eux-mêmes. Elles sont discrètes quant à leurs convictions religieuses mais ont à cœur de ne pas tromper leurs nouveaux amis.

La guerre remet en cause leur travail social. Après les grandes rafles de 1942, elles secondent le P. Démann, père de Sion, juif converti originaire d'Autriche-Hongrie et qui aide un grand nombre de juifs à se soustraire des rafles, sans intention de convertir ses protégés. Au lendemain de la guerre, après avoir vu de près les ravages de la Shoah, elles revisitent une fois encore leur apostolat envers les juifs qui devient plutôt une sorte de compagnonnage dans lequel la conversion n'est plus le but ultime, elle est remplacée par une compréhension mutuelle. Le témoignage d'une vie chrétienne en milieu juif prime. Cette évolution s'accompagne de conflits, avec la congrégation mère, qui se concluent par une rupture. La congrégation de Notre-Dame de Sion se maintient mais les Ancelles disparaissent dans les années 1960 lorsque les sœurs, à leur tour, après l'affaire Finaly et les décisions du Concile du Vatican, abandonnent toute idée de conversion des juifs. 
En plus de l'exposé clair et méthodique de cette transformation, O. Rota brosse un tableau de la présence des Ancelles en Europe (Rome, Anvers, Budapest), dans le monde (Montréal, Tunis, Fez) et bien sûr en Israël où les sœurs de Sion sont ellesmêmes présentes à Jérusalem.

À travers ce parcours des Ancelles de Notre-Dame de Sion, c'est, finalement, la mutation de l'apostolat catholique qui est finement analysée dans ce livre, bien renseigné : les références bibliographiques et archivistiques abondent dans les notes en bas de pages ; les fonds de la congrégation de Notre-Dame de Sion ont été largement fouillés et minutieusement exploités. L'ouvrage s'inscrit donc dans une histoire générale de l'Église catholique et son renoncement à un antisémitisme religieux que les Ancelles ont, de manière pionnière, mis en pratique.

Il faut remercier $\mathrm{O}$. Rota d'avoir mis en lumière leur présence dans cette métamorphose de la compréhension de l'apostolat envers les juifs. Les travaux sur le renouveau apostolique de l'Église ont laissé le rôle des Ancelles dans l'ombre alors qu'elles ont innové dans ce changement. 